\title{
Studying Signal Transduction in Single Dendritic Spines
}

\section{Ryohei Yasuda}

Neurobiology Department, Howard Hughes Medical Institute, Duke University Medical Center, Durham, North Carolina 27710

Correspondence: yasuda@neuro.duke.edu

\begin{abstract}
Many forms of synaptic plasticity are triggered by biochemical signaling that occurs in small postsynaptic compartments called dendritic spines, each of which typically houses the postsynaptic terminal associated with a single glutamatergic synapse. Recent advances in optical techniques allow investigators to monitor biochemical signaling in single dendritic spines and thus reveal the signaling mechanisms that link synaptic activity and the induction of synaptic plasticity. This is mostly in the study of $\mathrm{Ca}^{2+}$-dependent forms of synaptic plasticity for which many of the steps between $\mathrm{Ca}^{2+}$ influx and changes to the synapse are now known. This article introduces the new techniques used to investigate signaling in single dendritic spines and the neurobiological insights that they have produced.
\end{abstract}

$F^{\text {ach neuron typically receives } 1000-10,000}$ Esynaptic inputs and sends information to an axon, which branches to produce a similar number of synaptic outputs. Most excitatory postsynaptic terminals are associated with dendritic spines, small protrusions emanating from the dendritic surface (Nimchinsky et al. 2002; Alvarez and Sabatini 2007). Each spine has a volume of $\sim 0.1$ femtoliter, and connects to the parent dendrite through a narrow neck, which acts as a diffusion barrier and compartmentalizes biochemical reactions. $\mathrm{Ca}^{2+}$ influx into spines initiates a cascade of biochemical signals leading to various forms of synaptic plasticity including long-term potentiation (LTP).

Because LTP in hippocampal CA1 pyramidal neurons is a cellular mechanism that may underlie long-term memory formation, the sig- nal transduction underlying LTP has been extensively studied by pharmacological and genetic methods (Bliss and Collingridge 1993; Derkach et al. 2007). It is now well established that LTP is induced by $\mathrm{Ca}^{2+}$ influx into dendritic spines through NMDA-type glutamate receptors (NMDARs), which induces the insertion of AMPA-type glutamate receptors (AMPARs) into the synapse, thereby increasing the sensitivity of the postsynaptic terminal to glutamate (Derkach et al. 2007; Kessels and Malinow 2009). An increase of release probability during LTP has also been reported (Enoki et al. 2009), and thus both pre- and postsynaptic mechanisms may contribute to LTP (Lisman and Raghavachari 2006).

Manipulations of signal transduction using specific pharmacological inhibitors or genetic

Editors: Morgan Sheng, Bernardo Sabatini, and Thomas C. Südhof

Additional Perspectives on The Synapse available at www.cshperspectives.org

Copyright (C) 2012 Cold Spring Harbor Laboratory Press; all rights reserved; doi: 10.1101/cshperspect.a005611

Cite this article as Cold Spring Harb Perspect Biol 2012;4:a005611 
R. Yasuda

perturbations have identified many signaling pathways that connect $\mathrm{Ca}^{2+}$ to LTP induction. For example, LTP requires the activation of many signaling proteins, including $\mathrm{Ca}^{2+} /$ calmodulin-dependent kinase II (CaMKII), extracellular signal-related kinase (ERK), Phoshoinositide 3 kinase (PI3K), protein kinase $\mathrm{A}$ and $\mathrm{C}$, and GTPases such as Ras, Rab, and Rho (Kennedy et al. 2005). The list is continually growing, and the hundreds of implicated proteins form a complex signaling network whose contribution to LTP is still unclear (Bromberg et al. 2008).

Signaling dynamics in neurons have traditionally been measured using biochemical analyses (Bromberg et al. 2008). However, the spatiotemporal resolution of conventional biochemistry is limited, restricting analysis to the time scale of many minutes and requiring the homogenization of tissue containing millions of synapses and other cellular elements. Furthermore, resolving synaptically induced changes in signaling by biochemical analysis typically requires stimulating many synapses at the same time, which may produce unintended effects, for instance, excitotoxicity or homeostatic plasticity.

The size of dendritic spines is similar to the resolution of an optical microscope, permitting the optical analysis of biochemical signaling in each dendritic spine (Svoboda and Yasuda 2006). In particular, the advent of two-photonbased FRET techniques and the development of appropriate fluorescent reporters of specific bio- chemical reactions (see below) have provided readouts for signal transduction with high spatiotemporal resolution in live brain tissue (Svoboda and Yasuda 2006; Yasuda 2006). This has provided detailed information about the dynamics of signal transduction in spines and dendrites, and insights into the molecular mechanisms of synaptic plasticity.

\section{MONITORING SIGNAL TRANSDUCTION IN LIVE CELLS USING FRET}

\section{FRET Imaging of Intracellular Signaling}

The dynamics of intracellular signaling have been studied using fluorescence resonance energy transfer (FRET) (Miyawaki 2003; Ni and Zhang 2010). FRET occurs when a donor and acceptor fluorophore are within nanometers of each other (Lakowicz 2006). The excitation energy of the donor is transferred to the acceptor owing to a dipole-dipole interaction, decreasing donor fluorescence and increasing acceptor fluorescence. The efficiency of FRET decreases sharply with the distance $(r)$ between the acceptor and the donor:

$$
Y_{\mathrm{FRET}}=1 /\left[1+\left(r / R_{0}\right)^{6}\right]
$$

where $R_{0}$ is the distance that gives $Y_{\mathrm{FRET}}=0.5$ (Fig. 1A) (Förster 1993; Lakowicz 2006). $R_{0}$ is called the Förster distance, because it was calculated by Förster in 1947 and found to be a
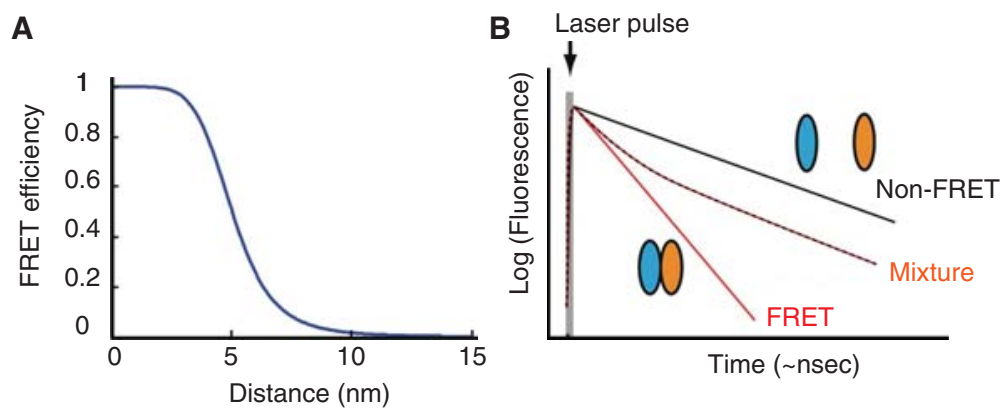

Figure 1. FRET for readout of signal transduction. (A) FRET efficiency as a function of the distance between donor and acceptor (when Förster distance $=5 \mathrm{~nm}$ ). (B) Fluorescence decay after short pulse excitation under non-FRET, FRET, and mixture conditions. 
function of several factors (Förster 1993; Lakowicz 2006):

$$
R_{0}=\left(8.79 \times 10^{17} J Q_{\mathrm{D}} \varepsilon_{\mathrm{A}} \kappa^{2} n^{-4}\right)^{1 / 6},
$$

where $J$ is the spectral overlap integral $J=\int f_{\mathrm{D}}$ $(\lambda) g_{\mathrm{A}}(\lambda) \lambda^{4} d \lambda$ (where $f_{\mathrm{D}}$ is the fluorescence spectrum of the donor normalized to the peak, $g_{\mathrm{A}}$ is the absorption spectrum of the acceptor normalized to the peak, and $\lambda$ is the wavelength), $\varepsilon_{\mathrm{A}}$ is the molar absorption coefficient of the acceptor, $Q_{D}$ is the quantum yield of donor, $\kappa^{2}$ is the orientation factor $(0 \leq \kappa \leq 4)$, and $n$ is the refractive index ( $\sim 1.3$ for solution). $\kappa^{2}$ depends on the relative orientation between the dipole moments of the donor emission and acceptor excitation. For random orientation, $\kappa^{2}=2 / 3$.

For good pairs of fluorescent donor and acceptor proteins such as enhanced cyan fluorescence protein (ECFP) and enhanced yellow fluorescent protein (EYFP) or enhanced green fluorescent protein (EGFP) and monomeric red fluorescent protein (mRFP), $R_{0}$ is $\sim 5 \mathrm{~nm}$. Because this distance is similar to the size of proteins, FRET can be used to detect intermolecular interactions between proteins tagged with fluorophores, or to detect intramolecular conformational changes of proteins tagged with both the donor and acceptor. Because Miyawaki et al. (1997) reported the first genetically encoded $\mathrm{Ca}^{2+}$ sensors based on FRET and fluorescent proteins, many FRET-based sensors designed to report the concentration of second messengers and the activity of protein kinases/phosphatases have been developed (Miyawaki 2003; $\mathrm{Ni}$ and Zhang 2010).

For imaging in biological systems, FRET is typically measured by taking the ratio of intensities of fluorescence of the donor and of the acceptor, which provides a fast and high-sensitivity measure of FRET (Lakowicz 2006). However, owing to the broadness of fluorophore absorption and excitation spectra, it is often impossible to excite the donor exclusively, or to separately measure donor and acceptor fluorescence (Wallrabe and Periasamy 2005; Lakowicz 2006). Because of this spectral bleedthrough, the ratio of donor and acceptor fluorescence depends on the ratio of fluorophore
Signal Transduction in Single Dendritic Spines

concentrations as well as on the FRETefficiency. Correcting for this concentration dependence is not simple and can severely complicate the analysis of FRET-based measurements. To avoid this problem, most FRET sensors designed for ratiometric imaging include both donor and acceptor in the same polypeptide, ensuring that the stoichiometry of the donor and acceptor is one to one (Miyawaki 2003; Ni and Zhang 2010).

FRET can also be measured by measuring the fluorescence lifetime of donor fluorophores using fluorescence lifetime imaging microscopy (FLIM) (Wallrabe and Periasamy 2005; Lakowicz 2006; Yasuda 2006). The fluorescence lifetime is the time elapsed from fluorophore excitation to photon emission, and is typically on the nanosecond scale. Because photon emission events occur stochastically, the fluorescence of a free donor after a short pulse excitation decays monoexponentially (Fig. 2) (Lakowicz 2006):

$$
F(t)=F_{0} \exp \left(-\mathrm{t} / \tau_{\mathrm{D}}\right)
$$

When FREToccurs, the fluorescent decay of the donor is accelerated because its excited state is now reduced by both photon emission and by FRET. The fluorescence lifetime of the donor bound to the acceptor, $\tau_{\mathrm{DA}}$, is related to the FRET efficiency $Y_{\text {FRET }}$ as (Lakowicz 2006)

$$
Y_{\mathrm{FRET}}=1-\tau_{\mathrm{DA}} / \tau_{\mathrm{D}}
$$

Because FLIM measures donor fluorescence only, it is not affected by changes in the concentrations of the donor and acceptor or by wavelength-dependent light scattering (Wallrabe and Periasamy 2005; Lakowicz 2006; Yasuda 2006). When the free donor and the donor bound to the acceptor coexist, the fluorescence decay becomes double exponential (Lakowicz 2006):

$$
\begin{aligned}
F(t)= & F_{0}\left[\mathrm{P}_{\mathrm{D}} \exp \left(-\mathrm{t} / \tau_{\mathrm{D}}\right)\right. \\
& +\mathrm{P}_{\mathrm{DA}} \exp \left(-\mathrm{t} / \tau_{\mathrm{DA}}\right),
\end{aligned}
$$

where $P_{\mathrm{D}}$ and $P_{\mathrm{DA}}$ are the proportions of donor that are free and bound to acceptor, respectively. Thus, one of the big advantages of fluorescence lifetime measurements is that the fraction of donor that is bound to acceptor is directly measured 
R. Yasuda

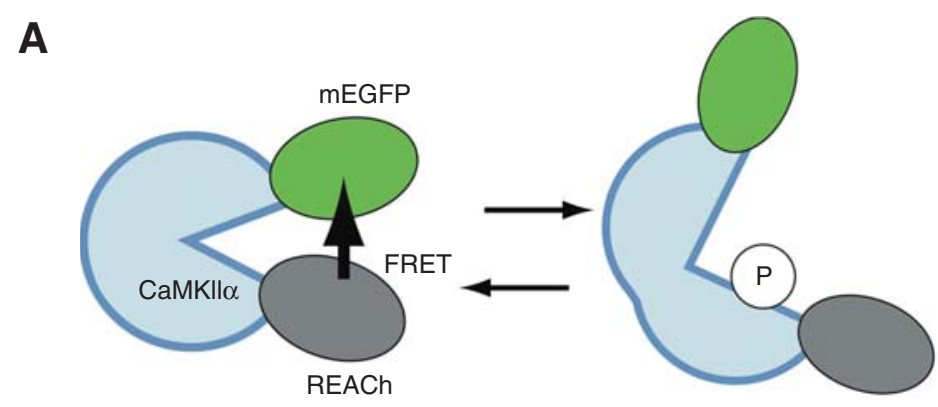

B Glutamate uncaging $(6 \mathrm{msec}, 0.5 \mathrm{~Hz})$

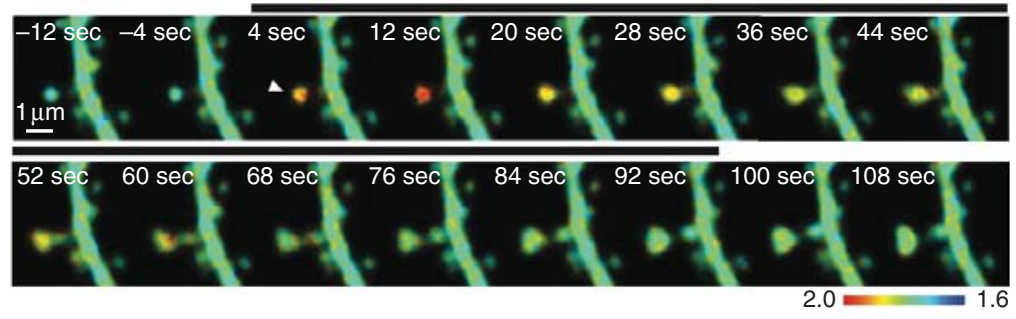

Figure 2. Imaging CaMKII activation during spine structural plasticity. (A) Schematic of the CaMKII sensor, Green-Camui $\alpha$. (B) CaMKII activation during spine enlargement triggered by stimulation of a single dendritic spine. (Figure adapted from Lee et al. 2009; reprinted, with permission, from Nature Publishing Group (C) 2009.)

by fitting the fluorescence decay curve with double exponential curves (Lakowicz 2006).

Several methods to image fluorescence lifetime have been developed (Lakowicz 2006). For example, time-correlated single-photon counting (TCSPC) uses a low light level, at which at most one photon is detected after each laser pulse (no photon after most laser pulses), and measures the timing between the excitation laser pulse and the detection of the emitted photon. The resulting histogram of the measured delays indicates the fluorescence decay (Lakowicz 2006). By combining this with scanning microscopy, one can obtain the fluorescence decay for each pixel in the image. One drawback of TCSPC is that accumulation of photons sufficient for making the histogram takes a relatively long time. After each photon detection, there is some dead time (approximately microseconds) in the instrumentation, and when the photon-counting rate is higher than the inverse of the dead time, the image can be saturated. To avoid this, one needs to decrease the excitation intensity to decrease photon-counting rates; thus, the system is optimal for dim samples in which one cannot waste any photons.

\section{Combining Two-Photon Laser Scanning FRET Imaging}

Two-photon laser scanning microscopy (2pLSM) enables imaging with high sensitivity and resolution in light scattering tissue (Denk et al. 1990; Svoboda and Yasuda 2006). Thus, combining FRET imaging techniques with 2pLSM allows monitoring of the biochemical state of small cellular compartments located deep within brain tissue (Svoboda and Yasuda 2006; Yasuda 2006). For this reason both 2pLSM ratiometric imaging and FLIM have been used to reveal the signaling cascades that act within dendritic spines to mediate LTP (see below).

Ratiometric FRET is accomplished in 2pLSM by splitting the fluorescence emitted by the donor and acceptor with dichroic mirrors and detecting the separated colors simultaneously by different photomultiplier tines (PMTs). It has been shown that FRET between 
CFP and YFP in single dendritic spines in brain slices can be imaged with two-color 2pLSM (Okamoto et al. 2004). Furthermore, because two-photon excitation of both fluorophores is achieved with a single laser and the image is built by laser scanning, the images of different color are automatically aligned.

More recently TCSPC was combined with 2pLSM (2pFLIM) to image FRET in single dendritic spines in brain slices with high sensitivity (Yasuda et al. 2006; Lee et al. 2009). Instrumentally the combination of 2PLSM and TCSPC is relatively straightforward, as both of them require a pulsed laser and scanning microscope (Yasuda 2006). The pulse rate of a typical modelock laser used for 2pLSM is $\sim 80 \mathrm{MHz}$, making the interpulse interval $(\sim 12 \mathrm{~ns})$ just long enough for measurements of fluorescence decay of typical fluorescent proteins $(2-4 \mathrm{~ns})$ after each pulse, while allowing measurements at close to the maximum repetition rate (Yasuda et al. 2006).

\section{Designing High-Sensitivity Sensors for FRET and FLIM}

The design criteria for FRET-based sensors are different for ratiometric imaging and FLIM (Yasuda et al. 2006). First, optimal donors for FLIM have monoexponential fluorescence decay, enabling easy calculation of binding fractions (Eq. 5). Although ECFP and its brighter variant Cerulean are the most common donors for ratiometric imaging, their fluorescence decay is multiexponential, making them unsuitable for FLIM. In contrast, EGFP and EYFP have monoexponential decays, making them ideal FLIM donors. As for acceptors, because ratiometric uses both donor and acceptor fluorescence, bright acceptors provide better signal-to-noise ratios. In contrast, FLIM measures donor fluorescence alone. Thus, the quantum yield of the acceptor can be very low, although the absorption coefficient needs to be high for high-yield FRET (Eq. 2). Thus, the recently developed resonance energy acceptor chromophore (REACh; a high-absorbing, dark EYFP variant), can be used as a FLIM acceptor (Ganesan et al. 2006; Murakoshi et al. 2008). Overall, whereas the
ECFP-EYFP pairs are widely used for ratiometric imaging, the EGFP-mRFP, EYFP-mRFP and EGFP-REACh pairs are used for FLIM (Tramier et al. 2006; Yasuda 2006; Murakoshi et al. 2008).

Most sensors developed so far use ECFPEYFP pairs and are optimized for ratiometric imaging (Miyawaki 2003; Ni and Zhang 2010). To convert them to FLIM sensors, one can simply replace the ECFP-EYFP pair with EGFPmRFP or EGFP-sREACh (Murakoshi et al. 2008). For example, the dynamics of actin polymerization in individual spines were first imaged by a ratiometric sensor (Okamoto et al. 2004). The sensor consists of ECFP-actin and Venus (a brighter variant of EYFP) -actin (Okamoto et al. 2004). When ECFP-actin and Venusactin copolymerize, FRET between CFP and Venus increases (Okamoto et al. 2004). Using this method, Okamoto et al. successfully used two-photon ratiometric imaging to visualize the polymerization of actin during spine structural changes induced by electric stimulation. Later the polymerization fraction was quantified with 2pFLIM in combination with a new actin polymerization sensor made of the mEGFP (monomeric EGFP: monomeric variant of EGFP) and sREACh (super REACh: high-folding REACh variant) pair (Murakoshi et al. 2008).

\section{ACTIVATION PROFILE OF SIGNALING MOLECULES MEASURED BY FRET IMAGING}

Two-Photon Photolysis to Stimulate Single Spines

To study signaling in single spines, it is necessary to trigger signaling events in a spatiotemporally controlled manner. The photolysis of caged compounds is one way to achieve this (Nerbonne et al. 1984; Walker et al. 1987; Li et al. 1998; Cai et al. 2004). In particular, because the development of MNI-glutamate, a caged glutamate with a high two-photon cross section, two-photon glutamate uncaging has been applied to study the physiology of single dendritic spines (Matsuzaki et al. 2001). Combining FRET imaging and two-photon glutamate encaging allows one to study the spatiotemporal 


\section{R. Yasuda}

dynamics of signal transduction in response to single spine stimulation (Harvey et al. 2008b; Lee et al. 2009).

\section{Compartmentalization of CaMKII Signaling}

$\mathrm{Ca}^{2+} /$ Calmodulin-dependent kinase II (CaMKII) is the most abundant protein in the postsynaptic density (Cheng et al. 2006), and is required for LTP (Silva et al. 1992; Giese et al. 1998; Lisman et al. 2002), spine structural plasticity (Matsuzaki et al. 2004; Lee et al. 2009; Pi et al. 2010), and some forms of learning and memory (Giese et al. 1998; Glazewski et al. 2000; Frankland et al. 2001). CaMKII is a holoenzyme consisting of 12 subunits, and each subunit acts as a serine/threonine kinase (Rosenberg et al. 2006). When $\left[\mathrm{Ca}^{2+}\right]$ increases, $\mathrm{Ca}^{2+} /$ Calmodulin binds to a CaMKII subunit (Braun and Schulman 1995; Lisman et al. 2002). This changes the structure of the CaMKII subunit from a closed form into an open form, exposing its kinase domain (Rosenberg et al. 2006; Chao et al. 2010). Activation of two adjacent subunits causes autophosphorylation of T286 (Lisman et al. 2002; Chao et al. 2010), which renders its activity independent of the interaction to $\mathrm{Ca}^{2+} / \mathrm{CaM}$, thereby keeping them activated after clearance of $\mathrm{Ca}^{2+}$ and dissociation of CaM (Saitoh and Schwartz 1985; Lou et al. 1986; Miller and Kennedy 1986; Yang and Schulman 1999). Because of this $\mathrm{Ca}^{2+}$-independent, autonomous activity of phosphorylated CaMKII, some speculated that CaMKII is a biochemical memory that lasts more than hours to maintain LTP (Lisman and Zhabotinsky 2001; Lisman et al. 2002).

The importance of T286 phorphorylation in LTP and learning was confirmed by experiments using mice with the autophosphorylation site mutated to alanine (T286A) (Giese et al. 1998; Hardingham et al. 2003). The presence of the persistent activity, however, has been controversial: Persistent T286 phosphorylation was observed biochemically following LTP (Fukunaga et al. 1995; Barria et al. 1997), whereas other studies showed that the $\mathrm{Ca}^{2+}$-independent activation of CaMKII decays much faster than T286 phosphorylation (Lengyel et al. 2004). Furthermore, pharmacological inhibition of CaMKII after establishing LTP does not typically affect the maintenance of LTP for most inhibitors (Malinow et al. 1989; Otmakhov et al. 1997; Chen et al. 2001), although one antagonist derived from a natural CaMKII inhibitor reversed LTP maintenance (Sanhueza et al. 2007).

To test the hypothesis of synapse-specific, persistent activity of CaMKII, the ratiometric CaMKII sensor Camui $\alpha$ was developed (Takao et al. 2005). Camui $\alpha$ consists of a CaMKII $\alpha$ subunit in which its ends are tagged with ECFP and Venus. FRET between ECFP and Venus reports the conformational change associated with its activation (Takao et al. 2005). However, the sensitivity was not sufficient for imaging in single spines in slices. More recently, the sensor was further optimized for 2pFLIM. In the new sensor called Green-Camui $\alpha$, the fluorophores were replaced to the pair of $\mathrm{mEGFP}$ and REACh (Ganesan et al. 2006; Murakoshi et al. 2008), which allowed measurement of CaMKII activity in single spines during LTP with high temporal resolution (approximately seconds) (Lee et al. 2009).

When a single spine was stimulated with two-photon glutamate uncaging to induce NMDAR-dependent LTP and associated spine enlargement, spine-specific CaMKII activation was observed (Lee et al. 2009). Contrary to the hypothesis that long-term autonomous CaMKII activity underlies the maintenance of LTP, CaMKII activity lasted only for $\sim 1 \mathrm{~min}$, whereas the plasticity lasted more than $1 \mathrm{~h}$ (Fig. 1). The study also clarified the impact of T286 phosphorylation as the activity of wild-type Camuix decays with the time constants of $6 \mathrm{sec}$ and $45 \mathrm{sec}$, whereas the T286A mutant is inactivated within $1 \mathrm{sec}$. Thus, T286 phosphorylation can underlie a biochemical memory for less than $1 \mathrm{~min}$, but not hours, allowing CaMKII to integrate brief $(\sim 0.1 \mathrm{sec})$ synaptic $\mathrm{Ca}^{2+}$ signals during repetitive stimulation (Sabatini et al. 2002; Noguchi et al. 2005).

\section{Spreading of Ras Signaling}

Ras is a small GTPase important for many forms of synaptic plasticity including LTP, synapse formation, spine structural plasticity, protein 
Signal Transduction in Single Dendritic Spines

synthesis in dendrites, and gene transcription (Wu et al. 2001; Zhu et al. 2002; Thomas and Huganir 2004). Because Ras-dependent signaling occurs in many different compartments, including spines, dendrites, and the nucleus, the spatiotemporal dynamics of Ras signaling are considered important in determining downstream effects (Yasuda et al. 2006). Thus, to fully understand the mechanism of Ras signaling, it is essential to measure the spatiotemporal dynamics of Ras activation.

The first Ras activation sensor, Raichu, was designed for ratiometric imaging and consists of CFP-RBD-Ras (without CAAX) -YFP-CAAX in a single polypeptide. RBD is a Ras-binding domain that binds to GTP-bound but not GDPbound Ras whereas CAAX is a membrane-targeted sequence derived from Ras itself (Mochizuki et al. 2001). Although this sensor worked in cell lines, it never was used for single synapse imaging. Also, the trafficking of this construct may be different from that of Ras. A next-generation Ras sensor, FRas, was designed specifically for 2pFLIM (Yasuda et al. 2006) and consists of $\mathrm{H}$-Ras tagged with monomeric enhanced green fluorescent protein (mEGFP) and the Ras-binding domain of Raf (RBD) tagged with two monomeric red fluorescence proteins ( $m R F P s)$. When mEGFP-Ras is activated, mRFP-RBD binds to mEGFP-Ras, thereby increasing FRET (Yasuda et al. 2006). Because RBD binding to Ras competed with Ras inactivation, the affinity between $\mathrm{RBD}$ and Ras was decreased by mutating the RBD (R59A), allowing fast reversal kinetics of the sensor (Yasuda et al. 2006). FRas-F in combination with 2pFLIM provided sufficient sensitivity to measure Ras activity in single spines in response to physiologically relevant stimuli (Yasuda et al. 2006; Harvey et al. 2008b). It was further confirmed that the activation kinetics measured by FRas-F are weakly correlated with expression level of Ras (Harvey et al. 2008b).

By combining 2pFLIM-based Ras imaging with two-photon glutamate uncaging, one can monitor Ras signaling in dendrites while stimulating a single spine (Harvey et al. 2008b). When the NMDARs on a single spine are opened, Ras activity increased in the stimulated spine, and rapidly spread into adjacent den- drites and spines (Harvey et al. 2008b). Ras activity in surrounding spines is close to $\sim 70 \%$ of that in the stimulated spine, but was not sufficient to induce plasticity. Because the expression of dominant-negative Ras or inhibition of the downstream kinase ERK causes inhibition of structural plasticity, these results showed that the Ras-ERK pathway is required, but not sufficient, for inducing structural plasticity. Pharmacological analysis indicated that the Ras-ERK pathway acts in parallel with the CaMKII pathway (Harvey et al. 2008b), which perhaps is required for the spine specificity of synaptic plasticity (Lee et al. 2009).

\section{MODELING SPATIOTEMPORAL DYNAMICS}

\section{Degree of Compartmentalization in Spines}

Once a protein is activated, the degree of compartmentalization is determined by competition between diffusion out of the spine and inactivation (Harvey et al. 2008b; Lee and Yasuda 2009). If the time constant of the spinedendrite diffusion coupling ( $\left.\tau_{\text {coupling }}\right)$ is much greater than the time constant of inactivation $\left(\tau_{\text {inactivation }}\right)$ (i.e., $\left.\tau_{\text {coupling }} \gg \tau_{\text {inactivation }}\right)$, the activity can be compartmentalized within the stimulated spine: In other words, the protein is inactivated before it spreads into the dendrite. However, this is not a sufficient condition for compartmentalized activity, because if an upstream inducer molecule spreads, the downstream effector molecule will show a diffuse pattern. Conversely, when $\tau_{\text {coupling }} \ll \tau_{\text {inactivation, }}$ the activity of the molecule should spread into dendrites.

$\tau_{\text {coupling }}$ depends on several morphological factors of spines:

$$
\tau_{\text {coupling }}=\frac{l V}{s D}
$$

where $V \sim 0.1 \mu \mathrm{m}^{3}$ is the volume of the spine, $l \sim 0.8 \mu \mathrm{m}$ is the spine neck length, $s \sim$ $0.008 \mu \mathrm{m}^{2}$ is the cross-section area of the spine neck, and $D$ is the effective diffusion constant of the molecule. For membrane proteins, $V \sim$ $1 \mu \mathrm{m}^{2}$ is the surface area of the spine and $s \sim$ 
R. Yasuda

$0.3 \mu \mathrm{m}$ is the circumference of the spine neck (Svoboda et al. 1996; Bloodgood and Sabatini 2005). Recently, several groups showed that $\tau_{\text {coupling }}$ is regulated in an activity-dependent manner (Bloodgood and Sabatini 2005; Grunditz et al. 2008). Thus, it is likely that synaptic activity regulates the degree of compartmentalization of biochemical signaling in dendritic spines.

The degree of compartmentalization has been measured for three molecules- $\mathrm{Ca}^{2+}$, CaMKII, and Ras-and all of these molecules follow the rules laid out (Lee and Yasuda, 2009). For example, $\tau_{\text {coupling }}$ for $\mathrm{Ca}^{2+}$ is about $100 \mathrm{~ms}$. By comparison, $\tau_{\text {inactivation, or in the }}$ case of $\mathrm{Ca}^{2+}$ - the extrusion time constant-is $\sim 20 \mathrm{~ms}$, which is much faster than $\tau_{\text {coupling }}$, producing compartmentalized $\mathrm{Ca}^{2+}$ elevation in spines in response to NMDAR activation ( $\mathrm{Sa}-$ batini et al. 2001). The spine-dendrite diffusion coupling for CaMKII is described with two time constants: $\tau_{\text {coupling }} \sim 1 \mathrm{~min}$ and $20 \mathrm{~min}$ (Okamoto et al. 2004; Lee et al. 2009). The inactivation also has two time constants: $\tau_{\text {inactivation }} \sim$ $5 \mathrm{sec}$ and $45 \mathrm{sec}$ (Lee et al. 2009). Thus, the law predicts that CaMKII is compartmentalized, as shown by the experimental results (Fig. 2) (Lee et al. 2009). On the other hand, Ras has slow inactivation kinetics with $\tau_{\text {inactivation }} \sim 200 \mathrm{sec}$ (Yasuda et al. 2006; Harvey et al. 2008b), whereas the diffusion is much faster, $\tau_{\text {coupling }} \sim 5 \mathrm{sec}$ (Harvey et al. 2008b). Thus, Ras shows a diffusive activity pattern (Fig. 3 ).

\section{Spatial Profile}

Once a molecule spreads into dendrite, it produces a specific pattern of activity that is dependent on its diffusion and inactivation constants and that can be modeled with a one-dimensional reaction-diffusion equation (Noguchi et al. 2005; Harvey et al. 2008b),

$$
\begin{aligned}
\frac{\partial \Phi(x, t)}{\partial t}= & D \frac{\partial^{2} \Phi(x, t)}{\partial x^{2}} \\
& -\frac{1}{\tau_{\text {inactivation }}} \Phi(x, t),
\end{aligned}
$$

where $\Phi$ is the spatiotemporal profile of activation, $D$ is the effective diffusion coefficient, and $\tau_{\text {inactivation }}$ is the time constant of inactivation. We assume that the protein is activated at a spine located at $x=0$, diffuses in the active state with diffusion constant $D$, and is inactivated at a rate given by $1 / \tau_{\text {inactivation. In this case, the }}$
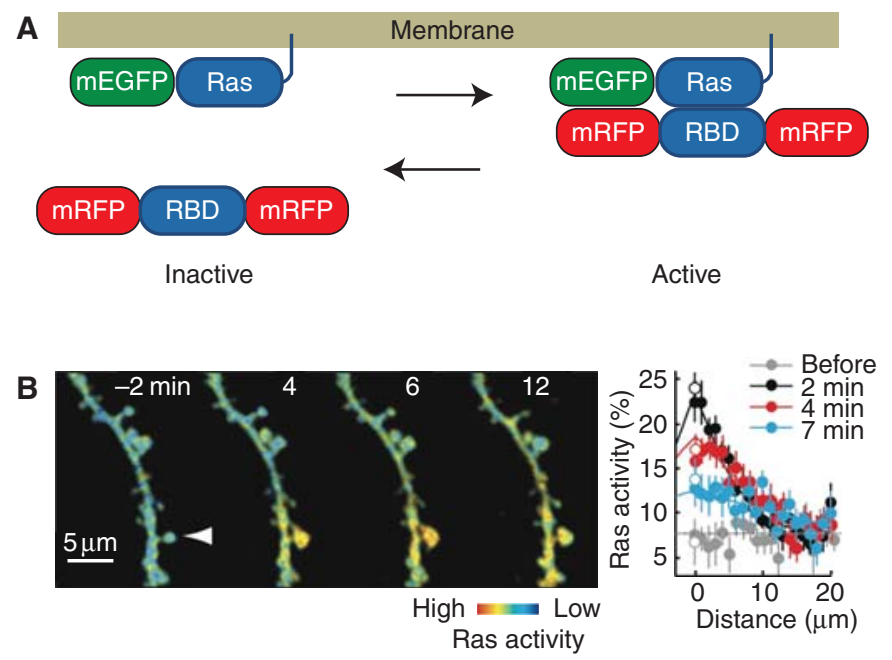

Figure 3. Imaging Ras activation during LTP. (A) Schematic of the Ras sensor. (B) Ras activation during LTP. (C) Spatial profile of Ras activation along the dendrite measured as a function of distance from the stimulated spine $(x=0)$. 
Signal Transduction in Single Dendritic Spines

steady-state solution is given by (Harvey et al. 2008b)

$$
\Phi=\exp (-x / L)
$$

where $L$ is the length constant given by

$$
L=\sqrt{D_{\tau_{\text {inactivation }}}} .
$$

Thus, the length constant of protein signaling is basically determined by two factors. For Ras, $D \sim 0.5 \mu \mathrm{m}^{2} / \mathrm{sec}$ (Lommerse et al. 2004; Murakoshi et al. 2004) and $\tau_{\text {inactivation }} \sim 200 \mathrm{sec}$ (Yasuda et al. 2006; Harvey et al. 2008b) yielding $L \sim 7 \mu \mathrm{m}$, consistent with the measured length constant (Fig. 3) (Harvey et al. 2008b). Overall, these approaches can be used to predict the spatial profile of signal transduction from the diffusion coefficient and inactivation time constant as long as the upstream signaling molecules do not spread (Harvey et al. 2008b; Lee and Yasuda 2009).

\section{SOURCE-SPECIFIC ACTIVATION OF SIGNALING MOLECULES VIA NANODOMAIN $\mathrm{Ca}^{2+}$}

$\mathrm{Ca}^{2+}$ nandomains, the region near the mouth of open $\mathrm{Ca}^{2+}$ channels where $\left[\mathrm{Ca}^{2+}\right]$ can be locally very high, have long been proposed to modulate signaling transduction (Neher 1998). The importance of nanodomain $\mathrm{Ca}^{2+}$ was shown by the different impacts of the $\mathrm{Ca}^{2+}$ chelators BAPTA and EGTA on the activation of $\mathrm{Ca}^{2+}$ dependent processes (Neher 1998; Augustine et al. 2003). Although BAPTA and EGTA have similar dissociation constants for $\mathrm{Ca}^{2+}$, BAPTA chelates $\mathrm{Ca}^{2+}$ ions 100 times faster than EGTA and can prevent $\mathrm{Ca}^{2+}$ ions from traveling more than a few angstroms by quickly capturing the ions after entry through the channel. In contrast, dependent on the chelator concentration, $\mathrm{Ca}^{2+}$ ions can travel many nanometers or even micrometers before being chelated by EGTA. Thus, BAPTA inhibits both global and nanodomain $\mathrm{Ca}^{2+}$ elevations, whereas EGTA selectively inhibits global $\mathrm{Ca}^{2+}$ elevations. It has been suggested that LTP is mediated via nanodomain signal transduction at specific subunits of NMDARs because, under some conditions, LTP induction has been found to be more sensitive to BAPTA and EGTA (Hoffman et al. 2002). Furthermore, pharmacological inhibition of NR2A and NR2B containing NMDARs can differentially impact LTP, suggesting that there may be subunit-specific signal transduction (Liu et al. 2004; Yashiro and Philpot 2008; Foster et al. 2010). Using CaMKII and Ras imaging techniques, we can now directly examine if the activation of these pathways is mediated via nanodomain signaling.

\section{CaMKII}

CaMKII interacts with many types of $\mathrm{Ca}^{2+}$ channels and transmitter receptors including L-type voltage-sensitive calcium channels (VSCCs) (Hudmon et al. 2005; Wheeler et al. 2008), T-type VSCCs (Welsby et al. 2003), P/Q-type VSCCs (Jiang et al. 2008), dopamine receptors (Liu et al. 2009), muscarinic receptors (Guo et al. 2010), and the NR2B subunit of NMDAR (Bayer et al. 2001; Merrill et al. 2005). This naturally leads to the hypothesis that opening of specific channels activates bound pools of CaMKII via nanodomain $\mathrm{Ca}^{2+}$. Indeed, CaMKII imaging in spines and dendrites supports this hypothesis (Lee et al. 2009). When a neuron is depolarized, CaMKII is activated in both spines and dendrites, with higher activation in dendrites. The spine but not dendritic CaMKII activation is prevented by the L-type channel inhibitor nimodipine. However, the same inhibitor does not reduce the depolarizationevoked $\mathrm{Ca}^{2+}$ elevation, suggesting that L-type channels do not contribute to global $\mathrm{Ca}^{2+}$ under this stimulus. Thus, CaMKII in spines can sense small calcium flow through L-type channels, but is not sensitive to large $\mathrm{Ca}^{2+}$ increases mediated by other VSCCs, consistent with nanodomain-mediated activation.

To confirm $\mathrm{Ca}^{2+}$-CaMKII nanodomain signaling, the sensitivity of CaMKII activation to BAPTA and EGTA was measured (Lee et al. 2009). When whole-cell recordings were obtained with an electrode containing $20 \mathrm{~mm}$ BAPTA, CaMKII activation in response to depolarization 
R. Yasuda

was inhibited. However, the same concentration of EGTA affected CaMKII activation to a smaller degree. Notably, unlike depolarization-induced CaMKII activation, glutamate uncaging-evoked CaMKII activation shows similar sensitivity to EGTA and BAPTA, suggesting that NMDAR-mediated CaMKII activation requires global $\mathrm{Ca}^{2+}$. Because CaMKII is not activated by high $\mathrm{Ca}^{2+}$ influx through non-L-type channels in response to depolarization, NMDARmediated CaMKII probably requires both nanodomain and global $\mathrm{Ca}^{2+}$.

The source-specific activation of CaMKII produces remarkable activation patterns during LTP induced by pairing postsynaptic depolarization and two-photon glutamate uncaging (Fig. 4) (Lee et al. 2009). The main purpose of the postsynaptic depolarization is to release $\mathrm{Mg}^{2+}$ block from NMDARs. However, depolarization also opens VSCCs, and produces large $\mathrm{Ca}^{2+}$ elevation in spines and dendrites whose amplitude can be larger than that owing to opening of NMDARs. In addition, whereas NMDAR $\mathrm{Ca}^{2+}$ lasts only for $\sim 0.1 \mathrm{sec}$, depolarization-induced $\mathrm{Ca}^{2+}$ is more prolonged, and lasts seconds until VSCCs are inactivated and $\mathrm{Ca}^{2+}$ decays. However, as discussed above, CaMKII activation in response to depolarization produces high CaMKII activity in dendrites, but much lower in spines (Fig. 4). Subsequent uncaging activates CaMKII only in the stimulated spine (Fig. 4), indicating that only a fraction of CaMKII can be stimulated by large, depolarization-induced $\mathrm{Ca}^{2+}$ increases. This is consistent with the fact that depolarization itself generally does not trigger LTP (Kullmann et al. 1992) even if, under some conditions, depolarization-induced CaMKII activation in spines can be sufficient to induce LTP in a synapse nonspecific manner (Kato et al. 2009).

\section{Ras}

Ras signaling is much more diffuse than CaMKII (Fig. 3), and thus source-specific signaling transduction may not occur. The relationship between global $\mathrm{Ca}^{2+}$ evoked by back-propagating action potentials and Ras activation in the dendrite has been measured-Ras activation is supralinearly related to $\mathrm{Ca}^{2+}$ elevation (Yasuda et al. 2006). However, when $\mathrm{Ca}^{2+}$ entry was reduced by pharmacological inhibition of specific classes of $\mathrm{Ca}^{2+}$ channels, the degree of Ras activation was independent of which channel was blocked and predicted by the amplitude of the remaining action potential-evoked $\mathrm{Ca}^{2+}$ increase (Fig. 5) (Yasuda et al. 2006). Hence, in contrast to CaMKII, Ras is activated by global $\mathrm{Ca}^{2+}$ and not by nanodomain $\mathrm{Ca}^{2+}$.

\section{THE FUNCTION OF SPREADING SIGNALS}

The differential spread of activity for each signaling molecule suggests that movement of molecules across the spine neck may have important functions. One possibility is that it is required for spine-to-dendrite signaling to bring resources from the adjacent dendrite into stimulated spines. For example, during chemical LTP induction and in response to a

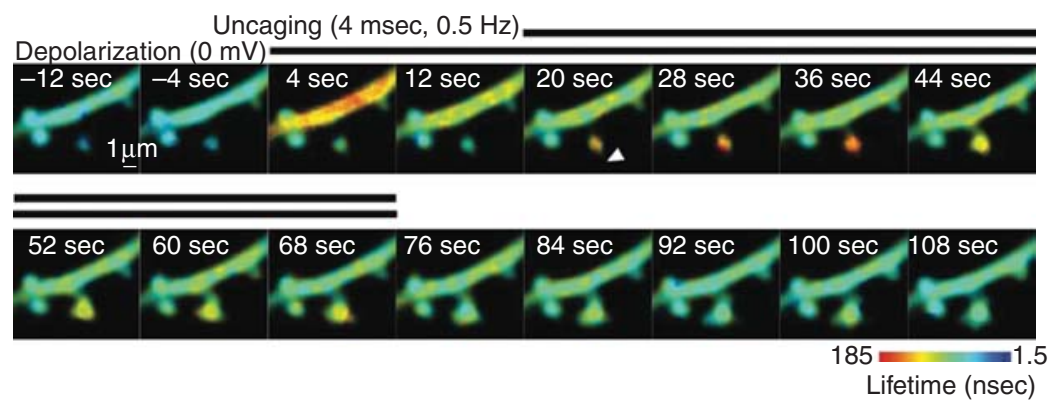

Figure 4. CaMKII activation during glutamate uncaging paired with depolarization. (Figure adapted from Lee et al. 2009; reprinted, with permission, from Nature Publishing Group (C) 2009.) 

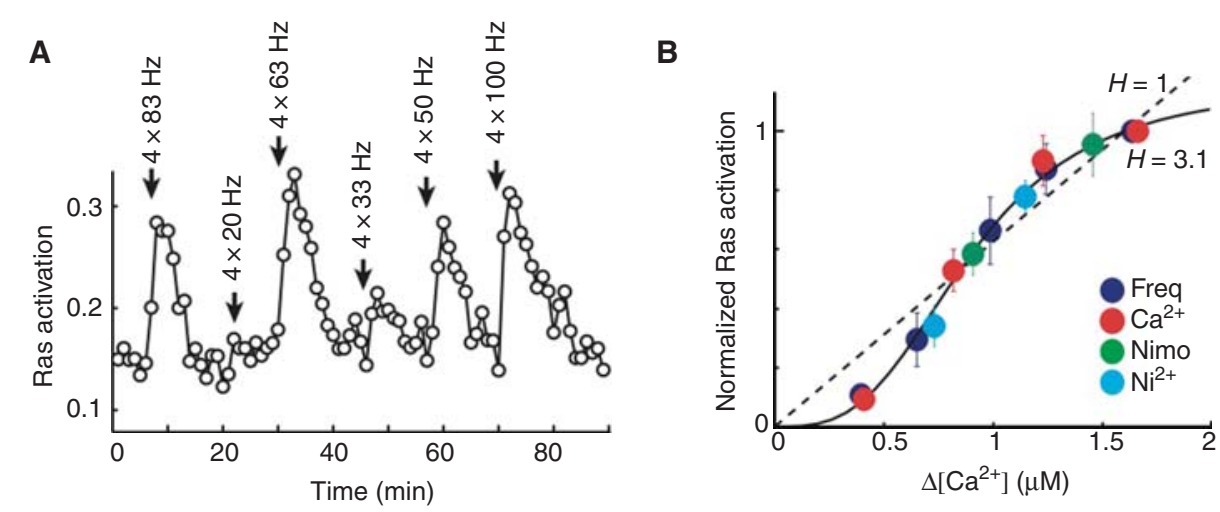

Figure 5. Supersensitive Ras activation as a function of $\mathrm{Ca}^{2+}$ concentration. (Image modified from Yasuda et al. 2006; reprinted, with permission, from Elsevier (C) 2006.)

signal that moves from spines to dendrites, endosomes containing transferrin receptors move from dendrites into spines (Park et al. 2004, 2006). In addition, AMPAR exocytosis during LTP occurs in dendrites near the stimulated spines as well as directly in the stimulated spines (Yudowski et al. 2007; Makino and Malinow 2009; Patterson et al. 2010). The exocytosis of AMPARs was imaged using superecliptic pHluorin, a pH-sensitive species of GFP. When the extracellular domain of the GluA1 subunit of AMPA receptor is in the endosome, fluorescence is quenched owing to low $\mathrm{pH}$ of that environment. This allows one to image surface AMPA receptors selectively (Yudowski et al. 2007; Makino and Malinow 2009; Patterson et al. 2010). Following photobleaching preexisting surface receptors, individual exocytosis events can be visualized as the appearance of new bright spots (Fig. 6). AMPAR exocytosis occurs over a $\sim 3 \mu \mathrm{m}$ stretch of dendrite near the stimulated spine, producing a similar spatial profile to that of the Ras pathway (Fig. 6) (Makino and Malinow 2009; Patterson et al. 2010). Activity-dependent AMPAR exocytosis is indeed Ras-ERK dependent (Patterson et al. 2010), suggesting that the spreading of Ras produces a similar spatial pattern in the downstream effects.

Another interesting possibility is that of heterosynaptic cross talk. Recently, a new form of plasticity that spans a similar length as activated Ras was described (Harvey and Svoboda 2007). Harvey and Svoboda (2007) stimulated a spine with a train of uncaging pulses that induces synaptic plasticity (suprathreshold stimuli). Within 1-5 min after the first stimulation, weak stimulation, which does not produce plasticity by itself (subthreshold stimuli), was applied to a neighboring dendritic spine less than $\sim 5 \mu \mathrm{m}$ away from the originally stimulated spine. This subthreshold stimulus was now sufficient to induce long-lasting synaptic plasticity. The spatiotemporal scale of the facilitation of plasticity is very similar to that of Ras activation, leading to the hypothesis that the spread of activated Ras causes this phenomenon. To test if Ras is required for this form of plasticity, Harvey and Svoboda perfused an inhibitor of signaling downstream from Ras (U0126) between the first, suprathreshold and second, subthreshold stimuli. This manipulation prevented the structural plasticity triggered by the second, subthreshold stimulus, but not to the first, suprathreshold stimulus (Harvey et al. 2008b). Furthermore, the subthreshold stimuli did not produce any additional Ras activation, suggesting that spreading of Ras resulting from the first stimulus was essential to produce the facilitation of plasticity.

\section{DISSECTING SIGNALING NETWORKS IN SINGLE DENDRITIC SPINES}

Imaging signal transduction in single dendritic spines has provided many insights into the biochemical mechanisms underlying synaptic plasticity. The same techniques promise to elucidate 
R. Yasuda

\section{A}

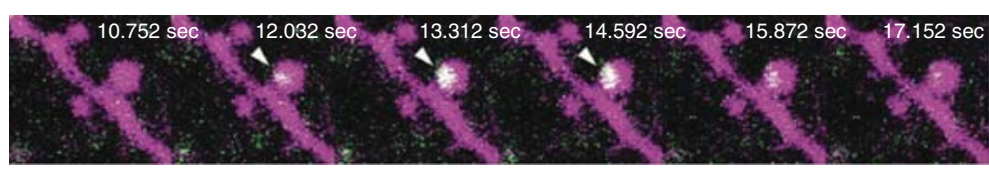

B

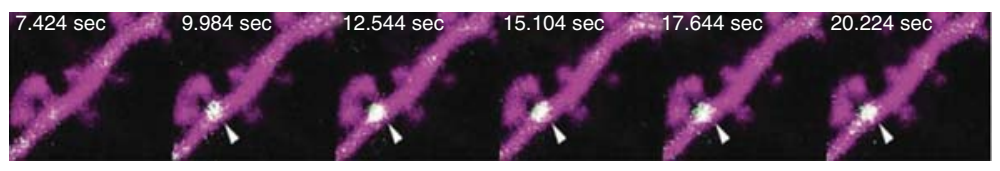

C

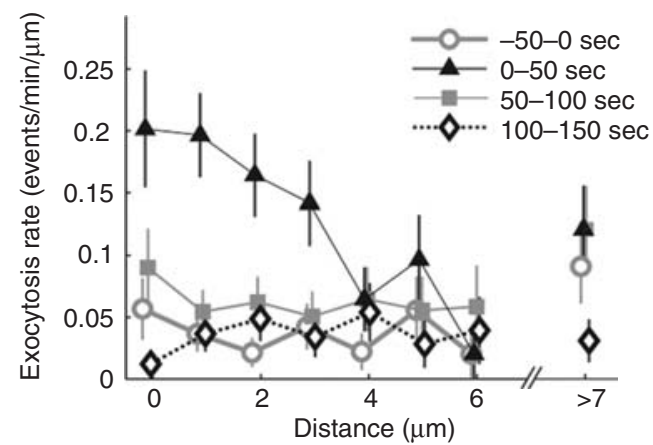

Figure 6. Spatiotemporal profile of AMPA receptor exocytosis. (A) Exocytosis in a stimulated spine. (B) Exocytosis in the dendrite. $(C)$ Spatial profile of AMPA receptor exocytosis (adapted from Patterson et al. 2010; reprinted, with permission, from the author and National Academy of Sciences (C) 2010).

the spatiotemporal profiles and functions of many other signaling pathways. In principle, we should be able to image all small GTPase proteins using sensors with a similar design to Ras, and recent work has examined Rho and CDC42 GTPases, which are also important for the morphogensis of spines and the function of synapses (Murakoshi et al. 2011). Also, because most kinases change their conformation when activated, a design similar to that used for CaMKII may work for many other kinases. A general design for kinase sensors based on substrate and phospho-binding domains ( $\mathrm{Ni}$ and Zhang 2010) also works for FLIM (Harvey et al. 2008a). Although it may not be possible to monitor the activation of all signaling proteins, combining pharmacological and genetic perturbation with FRETFLIM imaging techniques will produce a detailed view of the signaling cascades that are active in situ (Harvey et al. 2008b; Lee et al. 2009). Furthermore, changes in protein interactions in
PSD—e.g., CaMKII binding to the NMDARare thought to be important for synaptic plasticity (Bayer et al. 2001, 2006) and will be amenable to analysis by FRET-FLIM imaging.

Another interesting and developing front is the optical activation and inactivation of intracellular signaling proteins. Genetically encoded photoactivatable GTPases have been realized by several groups (Machacek et al. 2009; Wu et al. 2009; Wang et al. 2010) and will allow testing of the sufficiency of GTPase activity for inducing specific cell responses. Further, by combining readout of signaling with FRET-FLIM and photoactivation/inactivation, the biochemical interactions between two signaling components can be directly measured with high spatiotemporal resolution (Wang et al. 2010). Thus, by developing more techniques to measure and manipulate signaling proteins optically, the mechanisms of signal transduction in dendritic spines will be much better understood. 


\section{REFERENCES}

Alvarez VA, Sabatini BL. 2007. Anatomical and physiological plasticity of dendritic spines. Annu Rev Neurosci 30: 79-97.

Augustine GJ, Santamaria F, Tanaka K. 2003. Local calcium signaling in neurons. Neuron 40: 331-346.

Barria A, Muller D, Derkach V, Griffith LC, Soderling TR. 1997. Regulatory phosphorylation of AMPA-type glutamate receptors by CaM-KII during long-term potentiation. Science 276: 2042-2045.

Bayer KU, De Koninck P, Leonard AS, Hell JW, Schulman H. 2001. Interaction with the NMDA receptor locks CaMKII in an active conformation. Nature 411: 801-805.

Bayer KU, LeBel E, McDonald GL, O’Leary H, Schulman H, De Koninck P. 2006. Transition from reversible to persistent binding of CaMKII to postsynaptic sites and NR2B. J Neurosci 26: 1164-1174.

Bliss TV, Collingridge GL. 1993. A synaptic model of memory: Long-term potentiation in the hippocampus. Nature 361: 31-39.

Bloodgood BL, Sabatini BL. 2005. Neuronal activity regulates diffusion across the neck of dendritic spines. Science 310: $866-869$.

Braun AP, Schulman H. 1995. The multifunctional calcium/ calmodulin-dependent protein kinase: From form to function. Annu Rev Physiol 57: 417-445.

Bromberg KD, Ma'ayan A, Neves SR, Iyengar R. 2008. Design logic of a cannabinoid receptor signaling network that triggers neurite outgrowth. Science 320: 903-909.

Cai X, Liang CW, Muralidharan S, Kao JP, Tang CM, Thompson SM. 2004. Unique roles of SK and Kv4.2 potassium channels in dendritic integration. Neuron 44: $351-364$.

Chao LH, Pellicena P, Deindl S, Barclay LA, Schulman H, Kuriyan J. 2010. Intersubunit capture of regulatory segments is a component of cooperative CaMKII activation. Nat Struct Mol Biol 17: 264-272.

Chen HX, Otmakhov N, Strack S, Colbran RJ, Lisman JE. 2001. Is persistent activity of calcium/calmodulin-dependent kinase required for the maintenance of LTP? J Neurophysiol 85: 1368-1376.

Cheng D, Hoogenraad CC, Rush J, Ramm E, Schlager MA Duong DM, Xu P, Wijayawardana SR, Hanfelt J, Nakagawa T, et al. 2006. Relative and absolute quantification of postsynaptic density proteome isolated from rat forebrain and cerebellum. Mol Cell Proteomics 5: 1158-1170.

Denk W, Strickler JH, Webb WW. 1990. Two-photon laser scanning fluorescence microscopy. Science 248: 73-76.

Derkach VA, Oh MC, Guire ES, Soderling TR. 2007. Regulatory mechanisms of AMPA receptors in synaptic plasticity. Nat Rev Neurosci 8: 101-113.

Enoki R, Hu YL, Hamilton D, Fine A. 2009. Expression of long-term plasticity at individual synapses in hippocampus is graded, bidirectional, and mainly presynaptic: Optical quantal analysis. Neuron 62: 242-253.

Förster VT. 1993. Intermolecular energy migration and fluorescence (translation of Förster, T., 1948). In Biological physics (ed. Mielczarek EV, Greenbaum E, Knox RS), pp. 183-221. American Institute of Physics, New York.
Foster KA, McLaughlin N, Edbauer D, Phillips M, Bolton A Constantine-Paton M, Sheng M. 2010. Distinct roles of NR2A and NR2B cytoplasmic tails in long-term potentiation. J Neurosci 30: 2676-2685.

Frankland PW, O’Brien C, Ohno M, Kirkwood A, Silva AJ. 2001. $\alpha$-CaMKII-dependent plasticity in the cortex is required for permanent memory. Nature 411: 309-313.

Fukunaga K, Muller D, Miyamoto E. 1995. Increased phosphorylation of $\mathrm{Ca}^{2+} /$ calmodulin-dependent protein kinase II and its endogenous substrates in the induction of long-term potentiation. J Biol Chem 270: 6119-6124.

Ganesan S, Ameer-Beg SM, Ng TT, Vojnovic B, Wouters FS. 2006. A dark yellow fluorescent protein (YFP)-based resonance energy-accepting chromoprotein (REACh) for Forster resonance energy transfer with GFP. Proc Natl Acad Sci 103: 4089-4094.

Giese KP, Fedorov NB, Filipkowski RK, Silva AJ. 1998. Autophosphorylation at Thr286 of the $\alpha$ calcium-calmodulin kinase II in LTP and learning. Science 279: 870-873.

Glazewski S, Giese KP, Silva A, Fox K. 2000. The role of $\alpha$ CaMKII autophosphorylation in neocortical experiencedependent plasticity. Nat Neurosci 3: 911-918.

Grunditz A, Holbro N, Tian L, Zuo Y, Oertner TG. 2008. Spine neck plasticity controls postsynaptic calcium signals through electrical compartmentalization. J Neurosci 28: $13457-13466$.

Guo ML, Fibuch EE, Liu XY, Choe ES, Buch S, Mao LM, Wang JQ. 2010. CaMKII $\alpha$ interacts with M4 muscarinic receptors to control receptor and psychomotor function. EMBO J 29: 2070-2081.

Hardingham N, Glazewski S, Pakhotin P, Mizuno K, Chapman PF, Giese KP, Fox K. 2003. Neocortical long-term potentiation and experience-dependent synaptic plasticity require $\alpha$-calcium/calmodulin-dependent protein kinase II autophosphorylation. J Neurosci 23: 4428-4436.

Harvey CD, Svoboda K. 2007. Locally dynamic synaptic learning rules in pyramidal neuron dendrites. Nature 450: $1195-1200$.

Harvey CD, Ehrhardt AG, Cellurale C, Zhong H, Yasuda R, Davis RJ, Svoboda K. 2008a. A genetically encoded fluorescent sensor of ERK activity. Proc Natl Acad Sci 105: 19264-19269.

Harvey CD, Yasuda R, Zhong H, Svoboda K. 2008b. The spread of Ras activity triggered by activation of a single dendritic spine. Science 321: 136-140.

Hoffman DA, Sprengel R, Sakmann B. 2002. Molecular dissection of hippocampal $\theta$-burst pairing potentiation. Proc Natl Acad Sci 99: 7740-7745.

Hudmon A, Schulman H, Kim J, Maltez JM, Tsien RW, Pitt GS. 2005. CaMKII tethers to L-type $\mathrm{Ca}^{2+}$ channels, establishing a local and dedicated integrator of $\mathrm{Ca}^{2+}$ signals for facilitation. J Cell Biol 171: 537-547.

Jiang X, Lautermilch NJ, Watari H, Westenbroek RE, Scheuer T, Catterall WA. 2008. Modulation of CaV2.1 channels by $\mathrm{Ca}^{2+} /$ calmodulin-dependent protein kinase II bound to the C-terminal domain. Proc Natl Acad Sci 105: $341-346$.

Kato HK, Watabe AM, Manabe T. 2009. Non-Hebbian synaptic plasticity induced by repetitive postsynaptic action potentials. J Neurosci 29: 11153-11160. 
R. Yasuda

Kennedy MB, Beale HC, Carlisle HJ, Washburn LR. 2005 Integration of biochemical signalling in spines. Nat Rev Neurosci 6: 423-434.

Kessels HW, Malinow R. 2009. Synaptic AMPA receptor plasticity and behavior. Neuron 61: 340-350.

Kullmann DM, Perkel DJ, Manabe T, Nicoll RA. 1992. $\mathrm{Ca}^{2+}$ entry via postsynaptic voltage-sensitive $\mathrm{Ca}^{2+}$ channels can transiently potentiate excitatory synaptic transmission in the hippocampus. Neuron 9: 1175-1183.

Lakowicz JR. 2006. Principles of fluorescence spectroscopy, 3rd ed. Plenum, New York.

Lee SJ, Yasuda R. 2009. Spatiotemporal regulation of signaling in and out of dendritic spines: CaMKII and Ras. Open Neurosci J 3: 117-127.

Lee SJ, Escobedo-Lozoya Y, Szatmari EM, Yasuda R. 2009. Activation of CaMKII in single dendritic spines during long-term potentiation. Nature 458: 299-304.

Lengyel I, Voss K, Cammarota M, Bradshaw K, Brent V, Murphy KP, Giese KP, Rostas JA, Bliss TV. 2004. Autonomous activity of CaMKII is only transiently increased following the induction of long-term potentiation in the rat hippocampus. Eur J Neurosci 20: 3063-3072.

Li W, Llopis J, Whitney M, Zlokarnik G, Tsien RY. 1998. Cellpermeant caged InsP3 ester shows that $\mathrm{Ca}^{2+}$ spike frequency can optimize gene expression. Nature 392: $936-$ 941.

Lisman J, Raghavachari S. 2006. A unified model of the presynaptic and postsynaptic changes during LTP at CA1 synapses. Sci STKE 2006: rell.

Lisman JE, Zhabotinsky AM. 2001. A model of synaptic memory: A CaMKII/PP1 switch that potentiates transmission by organizing an AMPA receptor anchoring assembly. Neuron 31: 191-201.

Lisman J, Schulman H, Cline H. 2002. The molecular basis of CaMKII function in synaptic and behavioural memory. Nat Rev Neurosci 3: 175-190.

Liu L, Wong TP, Pozza MF, Lingenhoehl K, Wang Y, Sheng M, Auberson YP, Wang YT. 2004. Role of NMDA receptor subtypes in governing the direction of hippocampal synaptic plasticity. Science 304: 1021-1024.

Liu XY, Mao LM, Zhang GC, Papasian CJ, Fibuch EE, Lan HX, Zhou HF, Xu M, Wang JQ. 2009. Activity-dependent modulation of limbic dopamine D3 receptors by CaMKII. Neuron 61: 425-438.

Lommerse PH, Blab GA, Cognet L, Harms GS, Snaar-Jagalska BE, Spaink HP, Schmidt T. 2004. Single-molecule imaging of the $\mathrm{H}$-ras membrane-anchor reveals domains in the cytoplasmic leaflet of the cell membrane. Biophys $J$ 86: $609-616$.

Lou LL, Lloyd SJ, Schulman H. 1986. Activation of the multifunctional $\mathrm{Ca}^{2+} /$ calmodulin-dependent protein kinase by autophosphorylation: ATP modulates production of an autonomous enzyme. Proc Natl Acad Sci 83: $9497-$ 9501.

Machacek M, Hodgson L, Welch C, Elliott H, Pertz O, Nalbant P, Abell A, Johnson GL, Hahn KM, Danuser G. 2009. Coordination of Rho GTPase activities during cell protrusion. Nature 461: 99-103.

Makino H, Malinow R. 2009. AMPA receptor incorporation into synapses during LTP: The role of lateral movement and exocytosis. Neuron 64: 381-390.
Malinow R, Schulman H, Tsien RW. 1989. Inhibition of postsynaptic PKC or CaMKII blocks induction but not expression of LTP. Science 245: 862-866.

Matsuzaki M, Ellis-Davies GC, Nemoto T, Miyashita Y, Iino M, Kasai H. 2001. Dendritic spine geometry is critical for AMPA receptor expression in hippocampal CA1 pyramidal neurons. Nat Neurosci 4: 1086-1092.

Matsuzaki M, Honkura N, Ellis-Davies GC, Kasai H. 2004. Structural basis of long-term potentiation in single dendritic spines. Nature 429: 761-766.

Merrill MA, Chen Y, Strack S, Hell JW. 2005. Activity-driven postsynaptic translocation of CaMKII. Trends Pharmacol Sci 26: 645-653.

Miller SG, Kennedy MB. 1986. Regulation of brain type II $\mathrm{Ca}^{2+} /$ calmodulin-dependent protein kinase by autophosphorylation: $\mathrm{A} \mathrm{Ca}^{2+}$-triggered molecular switch. Cell 44: 861-870.

Miyawaki A. 2003. Visualization of the spatial and temporal dynamics of intracellular signaling. Dev Cell 4: 295-305.

Miyawaki A, Llopis J, Heim R, McCaffery JM, Adams JA, Ikura M, Tsien RY. 1997. Fluorescent indicators for $\mathrm{Ca}^{2+}$ based on green fluorescent proteins and calmodulin. $\mathrm{Na}$ ture 388: $882-887$.

Mochizuki N, Yamashita S, Kurokawa K, Ohba Y, Nagai T, Miyawaki A, Matsuda M. 2001. Spatio-temporal images of growth-factor-induced activation of Ras and Rap1. Nature 411: 1065-1068.

Murakoshi H, Ino R, Kobayashi T, Fujiwara T, Ohshima C, Yoshimura A, Kusumi A. 2004. Single-molecule imaging analysis of Ras activation in living cells. Proc Natl Acad Sci 101: 7317-7322

Murakoshi H, Lee S-J, Yasuda R. 2008. Highly sensitive and quantitative FRET-FLIM imaging in single dendritic spines using improved non-radiative YFP. Brain Cell Biol 36: 31-42.

Murakoshi H, Wang H, Yasuda R. 2011. Local, persistent activation of Rho GTPases during plasticity of single dendritic spines. Nature 472: 100-104.

Neher E. 1998. Usefulness and limitations of linear approximations to the understanding of $\mathrm{Ca}^{2+}$ signals. Cell Calcium 24: 345-357.

Nerbonne JM, Richard S, Nargeot J, Lester HA. 1984. New photoactivatable cyclic nucleotides produce intracellular jumps in cyclic AMP and cyclic GMP concentrations. Nature 310: 74-76.

Ni Q, Zhang J. 2010. Dynamic visualization of cellular signaling. Adv Biochem Eng Biotechnol 119: 79-97.

Nimchinsky EA, Sabatini BL, Svoboda K. 2002. Structure and function of dendritic spines. Annu Rev Physiol 64: 313-353.

Noguchi J, Matsuzaki M, Ellis-Davies GC, Kasai H. 2005. Spine-neck geometry determines NMDA receptor-dependent $\mathrm{Ca}^{2+}$ signaling in dendrites. Neuron 46: 609622.

Okamoto K, Nagai T, Miyawaki A, Hayashi Y. 2004. Rapid and persistent modulation of actin dynamics regulates postsynaptic reorganization underlying bidirectional plasticity. Nat Neurosci 7: 1104-1112.

Otmakhov N, Griffith LC, Lisman JE. 1997. Postsynaptic inhibitors of calcium/calmodulin-dependent protein kinase type II block induction but not maintenance of 
pairing-induced long-term potentiation. J Neurosci 17: 5357-5365.

Park M, Penick EC, Edwards JG, Kauer JA, Ehlers MD. 2004. Recycling endosomes supply AMPA receptors for LTP. Science 305: 1972-1975.

Park M, Salgado JM, Ostroff L, Helton TD, Robinson CG, Harris KM, Ehlers MD. 2006. Plasticity-induced growth of dendritic spines by exocytic trafficking from recycling endosomes. Neuron 52: 817-830.

Patterson MA, Szatmari EM, Yasuda R. 2010. AMPA receptors are exocytosed in stimulated spines and adjacent dendrites in a Ras-ERK-dependent manner during long-term potentiation. Proc Natl Acad Sci 107: 1595115956.

Pi HJ, Otmakhov N, El Gaamouch F, Lemelin D, De Koninck P, Lisman J. 2010. CaMKII control of spine size and synaptic strength: Role of phosphorylation states and nonenzymatic action. Proc Natl Acad Sci 107: 14437-14442.

Rosenberg OS, Deindl S, Comolli LR, Hoelz A, Downing KH, Nairn AC, Kuriyan J. 2006. Oligomerization states of the association domain and the holoenyzme of $\mathrm{Ca}^{2+} /$ CaM kinase II. FEBS J 273: 682-694.

Sabatini BL, Maravall M, Svoboda K. 2001. $\mathrm{Ca}^{2+}$ signaling in dendritic spines. Curr Opin Neurobiol 11: 349-356.

Sabatini BL, Oertner TG, Svoboda K. 2002. The life cycle of $\mathrm{Ca}^{2+}$ ions in dendritic spines. Neuron 33: 439-452.

Saitoh T, Schwartz JH. 1985. Phosphorylation-dependent subcellular translocation of a $\mathrm{Ca}^{2+} /$ calmodulin-dependent protein kinase produces an autonomous enzyme in Aplysia neurons. J Cell Biol 100: 835-842.

Sanhueza M, McIntyre CC, Lisman JE. 2007. Reversal of synaptic memory by $\mathrm{Ca}^{2+} /$ calmodulin-dependent protein kinase II inhibitor. J Neurosci 27: 5190-5199.

Silva AJ, Stevens CF, Tonegawa S, Wang Y. 1992. Deficient hippocampal long-term potentiation in $\alpha$-calcium-calmodulin kinase II mutant mice. Science 257: 201-206.

Svoboda K, Yasuda R. 2006. Principles of two-photon excitation microscopy and its applications to neuroscience. Neuron 50: 823-839.

Svoboda K, Tank DW, Denk W. 1996. Direct measurement of coupling between dendritic spines and shafts. Science 272: $716-719$.

Takao K, Okamoto K, Nakagawa T, Neve RL, Nagai T, Miyawaki A, Hashikawa T, Kobayashi S, Hayashi Y. 2005 Visualization of synaptic $\mathrm{Ca}^{2+} /$ calmodulin-dependent protein kinase II activity in living neurons. J Neurosci 25: 3107-3112.

Thomas GM, Huganir RL. 2004. MAPK cascade signalling and synaptic plasticity. Nat Rev Neurosci 5: 173-183.

Tramier M, Zahid M, Mevel JC, Masse MJ, Coppey-Moisan M. 2006. Sensitivity of CFP/YFP and GFP/mCherry
Signal Transduction in Single Dendritic Spines

pairs to donor photobleaching on FRET determination by fluorescence lifetime imaging microscopy in living cells. Microsc Res Tech 69: 933-939.

Walker JW, Somlyo AV, Goldman YE, Somlyo AP, Trentham DR. 1987. Kinetics of smooth and skeletal muscle activation by laser pulse photolysis of caged inositol 1,4,5-trisphosphate. Nature 327: 249-252.

Wallrabe H, Periasamy A. 2005. Imaging protein molecules using FRET and FLIM microscopy. Curr Opin Biotechnol 16: $19-27$.

Wang X, He L, Wu YI, Hahn KM, Montell DJ. 2010. Lightmediated activation reveals a key role for Rac in collective guidance of cell movement in vivo. Nat Cell Biol 12: 591-597.

Welsby PJ, Wang H, Wolfe JT, Colbran RJ, Johnson ML, Barrett PQ. 2003. A mechanism for the direct regulation of T-type calcium channels by $\mathrm{Ca}^{2+} /$ calmodulin-dependent kinase II. J Neurosci 23: 10116-10121.

Wheeler DG, Barrett CF, Groth RD, Safa P, Tsien RW. 2008. CaMKII locally encodes L-type channel activity to signal to nuclear CREB in excitation-transcription coupling. J Cell Biol 183: 849-863.

Wu GY, Deisseroth K, Tsien RW. 2001. Spaced stimuli stabilize MAPK pathway activation and its effects on dendritic morphology. Nat Neurosci 4: 151-158.

Wu YI, Frey D, Lungu OI, Jaehrig A, Schlichting I, Kuhlman B, Hahn KM. 2009. A genetically encoded photoactivatable Rac controls the motility of living cells. Nature 461: 104-108.

Yang E, Schulman H. 1999. Structural examination of autoregulation of multifunctional calcium/calmodulin-dependent protein kinase II. J Biol Chem 274: 2619926208.

Yashiro K, Philpot BD. 2008. Regulation of NMDA receptor subunit expression and its implications for LTD, LTP, and metaplasticity. Neuropharmacology 55: 1081-1094.

Yasuda R. 2006. Imaging spatiotemporal dynamics of neuronal signaling using fluorescence resonance energy transfer and fluorescence lifetime imaging microscopy. Curr Opin Neurobiol 16: 551-561.

Yasuda R, Harvey CD, Zhong H, Sobczyk A, van Aelst L, Svoboda K. 2006. Super-sensitive Ras activation in dendrites and spines revealed by 2-photon fluorescence lifetime imaging. Nat Neurosci 9: 283-291.

Yudowski GA, Puthenveedu MA, Leonoudakis D, Panicker S, Thorn KS, Beattie EC, von Zastrow M. 2007. Real-time imaging of discrete exocytic events mediating surface delivery of AMPA receptors. J Neurosci 27: 11112-11121.

Zhu JJ, Qin Y, Zhao M, Van Aelst L, Malinow R. 2002. Ras and Rap control AMPA receptor trafficking during synaptic plasticity. Cell 110: 443-455. 


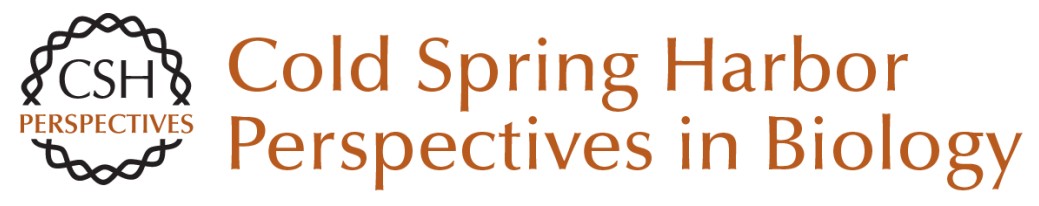

\section{Studying Signal Transduction in Single Dendritic Spines}

Ryohei Yasuda

Cold Spring Harb Perspect Biol 2012; doi: 10.1101/cshperspect.a005611 originally published online July 25, 2012

\section{Subject Collection The Synapse}

Studying Signal Transduction in Single Dendritic Spines Ryohei Yasuda

Synaptic Vesicle Pools and Dynamics AbdulRasheed A. Alabi and Richard W. Tsien

Synapses and Memory Storage Mark Mayford, Steven A. Siegelbaum and Eric R. Kandel

Synapses and Alzheimer's Disease Morgan Sheng, Bernardo L. Sabatini and Thomas C. Südhof

\section{Synaptic Cell Adhesion}

Markus Missler, Thomas C. Südhof and Thomas Biederer

Synaptic Dysfunction in Neurodevelopmental Disorders Associated with Autism and Intellectual Disabilities

Huda Y. Zoghbi and Mark F. Bear

The Postsynaptic Organization of Synapses Morgan Sheng and Eunjoon Kim

Presynaptic LTP and LTD of Excitatory and Inhibitory Synapses

Pablo E. Castillo
Synaptic Vesicle Endocytosis

Yasunori Saheki and Pietro De Camilli

Short-Term Presynaptic Plasticity Wade G. Regehr

NMDA Receptor-Dependent Long-Term

Potentiation and Long-Term Depression

(LTP/LTD) Christian Lüscher and Robert C. Malenka

Ultrastructure of Synapses in the Mammalian

Brain Kristen M. Harris and Richard J. Weinberg

Calcium Signaling in Dendritic Spines Michael J. Higley and Bernardo L. Sabatini

Synaptic Neurotransmitter-Gated Receptors Trevor G. Smart and Pierre Paoletti

Synaptic Vesicle Exocytosis

Thomas C. Südhof and Josep Rizo

Vesicular and Plasma Membrane Transporters for Neurotransmitters

Randy D. Blakely and Robert H. Edwards

For additional articles in this collection, see http://cshperspectives.cshlp.org/cgi/collection/

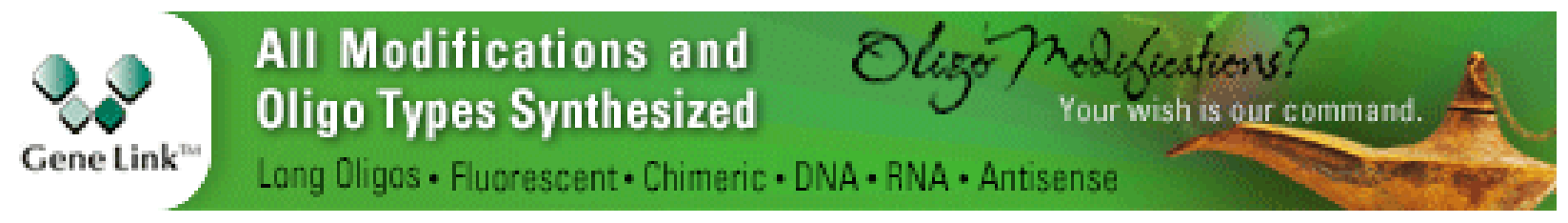

